\section{Neuer Prostatasymptomenscore: Ein Bild sagt mehr als tausend Worte}

Der International Prostate Symptom Score soll die subjektiven Beschwerden von Patienten mit Prostataleiden abschätzen. Doch was hilft ein Fragebogen, wenn der Patient inn nicht versteht und deshalb falsche Angaben macht? Eine Befragung im Bildformat soll dies nun ändern.

$\mathrm{D}$ er International Prostate Symptom Score (IPSS) soll subjektive Beschwerden in objektiven Zahlen auszudrücken, um das Leiden des Patienten zu quantifizieren. Doch in manchen Fällen führen falsche Score-Ergebnisse zu Fehlbeurteilungen. Am häufigsten missverstehen Patienten die Fragen 2 und 4 zur Häufigkeit und Dringlichkeit des Wasserlassens. Zudem gibt es ungenaue Übersetzungen des Fragebogens in die Landessprache des Patienten. Mancher Senior scheitert schlichtweg an der kleinen Schrift.

Abhilfe soll künftig der Visual Prostate Symptom Score (VPSS) schaffen. Er umfasst vier Fragen im Bildformat zu Häufigkeit des Wasserlassens am Tag, in der Nacht, Stärke des Urinstrahls und die ohnehin im Score bislang vermisste Frage nach der Lebensqualität. Die Ergebnisse des IPSS und des VPSS wurden auf der
Basis objektiver Messungen wie Urinflussrate und Restharnvolumen miteinander verglichen. Während nur $53 \%$ der 96 Männer den IPSS-Fragebogen ohne Hilfe ausfüllen konnten, waren beim VPSS immerhin $83 \%$ dazu in der Lage. Je niedriger die Schulbildung der Männer allerdings war, desto häufiger kam es zu Missverständnissen. So benötigten beim Ausfüllen des IPSS $87 \%$ der Probanden mit niedrigem Bildungsniveau Hilfe, Patienten aus dem höheren Bildungsbereich dagegen nur zu $24 \%$. Leichter verständlich für alle war offenbar der VPSS: Hier war Assistenz nur in $32 \%$ beziehungsweise $8 \%$ der Fälle nötig. Die Untersuchungen bestätigten zudem einen signifikante Korrelation zwischen VPSS und den Flussparametern, GesamtVPSS und IPSS sowie den einzelnen der vier VPSS-Parameter und ihren entsprechenden IPSS-Komponenten.

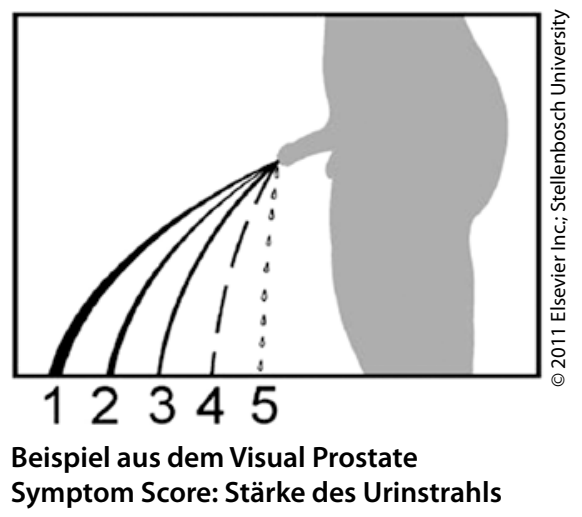

Fazit: Die Autoren sehen vor allem für Patienten mit niedrigem Bildungsniveau oder in Entwicklungsländern mit vielen Analphabeten klare Vorteile für den Bild-Score. Durch die reine Bildsprache können über alle Sprach- und Bildungsbarrieren hinweg die subjektiven Symptome bei Prostataproblemen einschließlich der Lebensqualität zuverlässig erfasst werden.

Dr. Christine Starostzik

van der Walt CLE et al. Prospective Comparison of a New Visual Prostate Symptom Score Versus the International Prostate Symptom Score in Men With Lower Urinary Tract Symptoms. Urology 2011; 78: 17-20

\title{
Inkontinenz nach Prostatektomie: Was bringt Beckenbodentraining?
}

Nach einer Prostataoperation leiden viele Männer an Harninkontinenz. Beckenbodentraining unter Anleitung eines Physiotherapeuten bringt zweierlei: Die Männer üben regelmäßiger, und es fließt mehr Geld in ihre Behandlung. Der Harnfluss ist aber hierbei nicht besser unter Kontrolle.

n zwei parallel durchgeführten, randomisierten Studien aus Großbritannien wurde untersucht, welchen Einfluss Beckenbodentraining auf die Kontinenz von Patienten nach radikaler Prostatektomie oder transurethraler Resektion der Prostata (TURP) hat. Das Training bestand aus vier Einzelsitzungen innerhalb von drei Monaten sowie Anleitungen für Übungen zuhause. Verglichen wurde mit der üblichen Versorgung, die lediglich Empfehlungen zum Lebensstil beinhaltete. Die Wirksamkeit wurde mit Fragebogen erhoben.

An Studie 1 beteiligten sich Männer, die sechs Wochen nach radikaler Prostatek- tomie inkontinent waren. Zwölf Monate nach dem Eingriff litten noch etwa drei Viertel von ihnen an Inkontinenz - unabhängig davon, ob sie ein individuelles Beckenbodentraining erhalten hatten oder nicht $(148 / 196=76 \%$ vs. $151 / 195=77 \%)$.

Studie 2 untersuchte Männer mit Inkontinenz infolge einer TURP sechs Wochen zuvor. Auch hier brachte das Einzeltraining keinen Vorteil, nach einem Jahr waren $65 \%$ $(126 / 194)$ und $62 \%(125 / 203)$ der Patienten noch nicht „trocken“.

In beiden Interventionsgruppen machten mehr Patienten (irgendwann) Übungen für ihren Beckenboden (67\% vs. $50 \%$ in
Studie $1 ; 55 \%$ vs. $20 \%$ in Studie 2). Durch die Intervention wurden zudem in beiden Studien mehr Kosten verursacht, im Mittel um $180 £$ oder $209 £$ pro Patient.

Fazit: Den Autoren zufolge sprechen die Studienergebnisse nicht gegen die Wirksamkeit des Beckenbodentrainings. Vielmehr brächte eine individuelle Beratung vor dem Hintergrund des vorhandenen Informationsangebots keinen Vorteil. Die Autoren räumen ein, dass die Datenlage für das Beckenbodentraining beim Mann lange nicht so gut sei wie bei der Frau. Gemäß den Cochrane-Reviews bestehe „ungenügende Evidenz für die Wirksamkeit“ dieser Maßnahme. Dr. Beate Schumacher

Glazener $\mathrm{C}$ et al. Urinary incontinence in men after formal one-to-one pelvic-floor muscle training following radical prostatectomy or transurethral resection of the prostate (MAPS): two parallel randomised controlled trials. Lancet 2011; 378: 328-37 